\title{
Antibiotic sensitivity pattern of Salmonella enterica serovar Typhi with special reference to nalidixic acid
}

\author{
Swarooparani N.B. ${ }^{1}$, Mita D. Wadekar ${ }^{2, *}$ \\ ${ }^{1}$ Associate Professor, ${ }^{2}$ Assistant Professor, Dept. of Microbiology, ${ }^{1}$ Subbaiah Institute of Medical Sciences, Shimoga, \\ Karnataka, ${ }^{2}$ Chamarajanagar Institute of Medical Sciences, Chamarajanagar, Karnataka, India
}

\section{*Corresponding Author:}

E-mail: drmdw20@gmail.com

\begin{abstract}
Introduction: Recently, Salmonella Typhi resistance to many of the commonly used antibiotics like chloramphenicol, amoxicillin and cotrimoxazole, has posed a challenge to treat enteric fever. Minimum inhibitory concentration (MIC) of ciprofloxacin is increasing which is responsible for both treatment failure and causing serious complications. Resistance to nalidixic acid can be used as an indirect indicator to detect increased ciprofloxacin MIC. Hence, study was conducted to know the antibiotic sensitivity pattern of Salmonella enterica serovar Typhi with special reference to nalidixic acid resistance.

Materials and Methods: A retrospective study of $S$. Typhi isolates was conducted. 106 blood samples from patients of all age group who were clinically suspected of enteric fever were included. Blood culture was done and $S$. Typhi isolates were identified and tested for susceptibility to ampicillin, chloramphenicol, cotrimoxazole, ciprofloxacin, ceftriaxone and nalidixic acid by disc diffusion method.

Result: 22(20.8\%) S. Typhi isolates were isolated from 106 blood samples. Chloramphenicol showed $100 \%$ sensitivity. Susceptibility to ampicillin (81.8\%), cotrimoxazole (81.8\%) and ceftriaxone (90.9\%) was high and to ciprofloxacin (72.7\%) and nalidixic acid $(45.5 \%)$ was low.

Conclusion: Ampicillin, chloramphenicol, cotrimoxazole and ceftriaxone showed maximum sensitivity and hence can be used for effective treatment. Nalidixic acid resistance was very high and all these resistant isolates showed decreased sensitivity to ciprofloxacin. Hence nalidixic acid sensitivity can be used for the detection of decreased susceptibility to ciprofloxacin.
\end{abstract}

Keywords: Salmonella Typhi, Ciprofloxacin, Resistance, Nalidixic acid.

\section{Introduction}

Enteric fever, caused by Salmonella spp., is an acute and generalized infection. ${ }^{1}$ It is a common health problem worldwide and is endemic in developing countries like India. Mortality rate (30\%) is high if not treated appropriately. ${ }^{2}$ Years ago, ampicillin, cotrimoxazole and chloramphenicol were the drug of choice for treatment. ${ }^{3}$ Unfortunately, resistance to these antibiotics has emerged. Resistance to three or more different groups of antibiotics simultaneously is known as multidrug resistant (MDR) Salmonella. ${ }^{2}$ Because of the increased percentage of MDR strains, ciprofloxacin and third generation cephalosporins (ceftriaxone) are in increase use. In 1990s, fluoroquinolone use was implemented which is a broad-spectrum antimicrobial agent which targets DNA gyrase. ${ }^{4}$ Recently, the number of strains of Salmonella spp. with decreased ciprofloxacin susceptibility has increased. ${ }^{5}$ Mechanism of action of resistance is mutation within the DNA gyrase with decreased outer membrane proteins expression and over expression of efflux pump. ${ }^{6}$ Treatment failure with ciprofloxacin has increased due to reduced susceptibility, especially with short course or low dose regimens. In such cases, either third generation cephalosporins (ceftriaxone) or chloramphenicol will be helpful. These isolates with reduced susceptibility appear susceptible with disc diffusion tests which are performed routinely. ${ }^{7,8}$ The increased emergence of MDR Salmonella spp. resulted in a need to develop newer antibiotics and also to find the mechanism by which they acquire resistance to available drugs. ${ }^{9}$ Nalidixic acid was considered to be the first synthetic quinolone antibiotics. Structure of it is similar to the newer generation quinolones. Mechanism of action of development of resistance is due to single point mutation in the quinolone resistancedetermining region (QRDR) of the gene gyrA. It also leads to decreased in vivo ciprofloxacin susceptibility. ${ }^{10}$ Number of studies have shown that resistance to nalidixic acid can be used to detect decreased sensitivity to ciprofloxacin. ${ }^{11,12}$ Strains showing decreased susceptibility to ciprofloxacin is responsible for treatment failure which is increasing in India and other countries. ${ }^{13,14}$ Such strains require higher concentrations of ciprofloxacin for inhibition. ${ }^{15}$ Hence, this study was conducted to know the antibiotic sensitivity pattern of Salmonella enterica serovar Typhi with special reference to nalidixic acid.

\section{Materials and Methods}

A retrospective analysis of blood culture results from clinically suspected cases of enteric fever was performed. The details of patients, isolated organism and the antimicrobial susceptibility patterns were collected from the registration records. The data was then analyzed by entering into Excel.

All blood samples from clinically suspected cases of enteric fever were sent for microbiological analysis. 
Blood samples were collected in brain heart infusion (BHI) broth and incubated at $37^{\circ} \mathrm{C}$ overnight. Subcultures were done on MacConkey agar and blood agar at days 1,2 and 5 of incubation to check for growth. Non lactose fermenting colonies grown were identified as Salmonella Typhi by standard biochemical reactions. ${ }^{16}$

Antimicrobial susceptibility tests were done on Mueller-Hinton agar (MHA) using Kirby-Bauer disk diffusion method. ${ }^{17}$ The antibiotics tested included: Ampicillin $(10 \mu \mathrm{g}), \quad$ chloramphenicol $(30 \mu \mathrm{g})$, cotrimoxazole $(1.25 \mu \mathrm{g} / 23.75 \mu \mathrm{g})$, ciprofloxacin $(5 \mu \mathrm{g})$, ceftriaxone $(30 \mu \mathrm{g})$ and nalidixic acid $(30 \mu \mathrm{g})$.
Resistance data were interpreted according to Clinical Laboratory Standards Institute. ${ }^{17}$

\section{Results}

Of 106 blood samples cultured, $S$. Typhi were isolated in 22(20.8\%) samples. Susceptible to first line drugs like ampicillin 18(81.8\%), chloramphenicol $22(100 \%)$, cotrimoxazole $18(81.8 \%)$ and also to ceftriaxone $20(90.9 \%)$ was high. But sensitivity to ciprofloxacin and nalidixic acid was very less. Only $10(45.5 \%)$ isolates in case of nalidixic acid and $16(72.7 \%)$ in ciprofloxacin were sensitive. All nalidixic acid resistant isolates were resistant to ciprofloxacin.

Table 1: Antibiotic sensitivity pattern of S. Typhi

\begin{tabular}{|c|c|c|c|c|c|c|}
\hline Organism & AMP & C & COT & CTR & CIP & NA \\
\hline $\begin{array}{c}\text { S. typhi } \\
\text { n=22 }\end{array}$ & 18 & 22 & 18 & 20 & 16 & 10 \\
$(81.8)$ & $(100)$ & $(81.8)$ & $(90.9)$ & $(72.7)$ & $(45.5)$ \\
\hline
\end{tabular}

AMP - Ampicillin, C - Chloramphenicol, COT - Cotrimoxazole, CTR - Ceftriaxone, CIP - Ciprofloxacin, NA Nalidixic acid

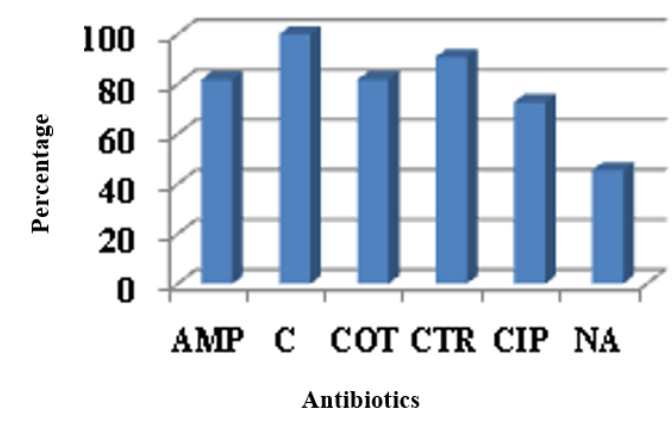

Fig. 1: Antibiotic sensitivity pattern of $S$. Typhi

\section{Discussion}

Enteric fever is a common problem in India. Several factors like poor sanitation and lack of better health care facilities contribute to it. ${ }^{18}$ Laboratory diagnosis can be done by isolation of organism by culture, serological detection of antigen and antibody. But definitive diagnosis requires the isolation of Salmonella spp. from clinical samples. ${ }^{19}$

In our study, 22(20.8\%) S. Typhi was isolated. This prevalence is similar to study done by Bulbul Hasan et al. ${ }^{20}$ Multi-drug resistant (MDR) Salmonella typhi showing resistance to ampicillin, chloramphenicol and co-trimoxazole is common and is often plasmid mediated. ${ }^{21}$ But with increased use of fluroquinolones and cephalosporins, it is declining. ${ }^{22}$ These MDR strains increases morbidity and mortality. Data in our study showed highest sensitivity to chloramphenicol (100\%) followed by ampicillin (81.8\%) and cotrimoxazole (81.8\%). Sensitivity pattern to these antibiotics indicates that MDR strains were very less but still exists in this region. These drugs (chloramphenicol, ampicillin and cotrimoxazole) can be used as first line antibiotics for the appropriate treatment of enteric fever. This finding is similar to study done by Lovely Akter et al. ${ }^{23}$ Our study also showed high sensitivity to ceftriaxone $(90.9 \%)$ which still remains the drug of choice. Similar highest sensitivity to ceftriaxone was seen in study done by B. A. Rahman et al. ${ }^{24}$ Ciprofloxacin $(72.7 \%)$ and nalidixic acid $(45.5 \%)$ sensitivity was very less, also the nalidixic acid-resistant isolates showed decreased susceptibility to ciprofloxacin. One of the studies done by Hakanen et al. ${ }^{25}$ showed the sensitivity and specificity of nalidixic acid susceptibility testing is good for screening of isolates with decreased ciprofloxacin susceptibility. Nalidixic acid resistance detection by disk diffusion method has led to the identification of all isolates for which the MICs of ciprofloxacin were $\geq 0.125 \mathrm{mg} / \mathrm{ml}$. Hence, it is interpreted that disc diffusion test to detect resistance to nalidixic acid, for fluoroquinolones, is reliable as it correlates well with the MIC values. ${ }^{26}$

\section{Conclusion}

The first-line antibiotics like ampicillin, chloramphenicol, cotrimoxozole and ceftriaxone can be used for the appropriate management of enteric fever. Resistance to quinolones is increasing and is of concern especially with respect to increase in its MIC levels. Nalidixic acid resistance can be used as a marker for predicting decreased sensitivity to ciprofloxacin. So any isolate showing resistance to nalidixic acid should be reported as intermediately susceptible to ciprofloxacin.

\section{References}

1. John A. Crump, Timothy J. Barrett, Jennifer T. Nelson, and Frederick J. Angulo. Reevaluating Fluoroquinolone Breakpoints for Salmonella enterica Serotype Typhi and for Non-Typhi Salmonellae. Clinical Infectious Diseases; 2003;37:75-81.

2. Gopal muthu, Arumugam suresh, Gnadesikan sumathy and Ramesh srivani. Studies on antimicrobial susceptibility pattern of Salmonella isolates from 
Chennai, India. International Journal of Pharma and Bio Sciences; 2011;2(2):B435-442.

3. S.M. Zahurul Haque Asna, J. Ashraful Haq and Md. Mushfequr Rahman. Nalidixic acid resistant Salmonella enterica Serotype Typhi with Decreased Susceptibility to Ciprofloxacin Caused Treatment Failure: A Report from Bangladesh. Jpn. J. Infect. Dis.; 2003;56:32-3.

4. K. E. Lee, J. H. Jung, B. Y. Jung, Y. H. Park AND Y. H. Lee. Characterization of Nalidixic Acid-Resistant and Fluoroquinolone-Reduced Susceptible Salmonella Typhimurium in Swine. Journal of Food Protection; 2011;74(4):610-5.

5. Marianne M. Lindgren, Pirkko Kotilainen, Pentti Huovinen, Saija Hurme, Susanna Lukinmaa, Mark A. Webber, Laura J.V. Piddock, Anja Siitonen, and Antti J. Hakanen. Reduced Fluoroquinolone Susceptibility in Salmonella enteric Isolates from Travelers, Finland. Emerging Infectious Diseases; 2009;15(5):809-12.

6. Kais Kassim Ghaima, Hiba Hazim Hamid, Hiba Muneer Al-Khafaji. Detection of qnr resistance genes in Ciprofloxacin and Nalidixic acid resistant Salmonella spp. isolated from stool samples. International Journal of Advanced Research; 2016;4(3):577-82.

7. Acharya D, Malla S, Bhatta DR, Adhikari N, Dumre SP. Current Fluoroquinolone Susceptibility Criteria for Salmonella Needs Re-evaluation. Kathmandu University Medical Journal; 2012;10(1):24-9.

8. Hayath Kownhar, Esaki Muthu Shankar, Ramachandran Rajan and Usha Anand Rao. Emergence of nalidixic acidresistant Salmonella enterica serovar Typhi resistant to ciprofloxacin in India. Journal of Medical Microbiology;2007:136-7.

9. Hafsa Afroz, Md. Manjur Hossain, Md. Fakruddin. A 6 year retrospective study of bloodstream Salmonella infection and antibiotic susceptibility of Salmonella enterica serovar Typhi and Paratyphi in a tertiary care hospital in Dhaka, Bangladesh. Tzu Chi Medical Journal; 2014;26:73-8.

10. Abbasi S, Imtiaz A, Usman J, Kaleem F, Hassan A. Evaluation of the current trend of nalidixic acid susceptibility in typhoidal Salmonellae; a marker of therapeutic failure for the fluoroquinolones. Iranian Journal of Microbiology; 2011;3(2):80-3.

11. Shyamapada Mandal, Manisha DebMandal, Nishith Kumar Pal. Nalidixic acid susceptibility status of Salmonella enterica serovar Typhi isolates from Kolkata, India. Jundishapur Journal of Microbiology;2011;4(1):5560.

12. Antti J. Hakanen, Marianne Lindgren, Pentti Huovinen, Jari Jalava, Anja Siitonen and Pirkko Kotilainen. New Quinolone Resistance Phenomenon in Salmonella enterica: Nalidixic Acid-Susceptible Isolates with Reduced Fluoroquinolone Susceptibility. Journal of Clinical Microbiology; 2005;43(11):5775-8.

13. Pallab Ray, Jyoti Sharma, Rungmei S.K. Marak and R.K. Garg. Predictive efficacy of nalidixic acid resistance as a marker of fluoroquinolone resistance in Salmonella enterica var Typhi. Indian J Med Res; 2006;124:105-8.

14. Kapil A, Das B. Nalidixic acid susceptibility to screen ciprofloxacin resistance in Salmonella typhi. Indian J Med Res;2002;115:49-54.

15. Basudha Khanal, Sanjib Kumar Sharma, Shyamal Kumar Bhattacharya, Narayan Raj Bhattarai, Monorama Deb, and Reba Kanungo. Antimicrobial Susceptibility Patterns of Salmonella enterica Serotype Typhi in Eastern Nepal. J Health Popul Nutr; 2007;25(1):82-7.
16. J.G. Collee, Barrie P. Marmion, AG Fraser, A. Simmons. 2007. Mackie and McCartney Practical Medical Microbio logy, $14^{\text {th }}$ ed. Edinburgh: Churchill Livingstone.

17. Clinical Laboratory Standards Institutes (CLSI). Performance Standards for antimicrobiasusceptibility testing, XXI International Supplement (M100-S27). Wayne, Penns ylvania, USA: National Committee for Clinical Laboratory Stand ards;2017.

18. Kavita Nagshetty, Shivannavar T. Channappa and Subhashchandra M. Gaddad. Antimicrobial susceptibility of Salmonella Typhi in India. J Infect Dev Ctries; 2010; 4(2): 070-073.

19. SP Kalra, N Naithani, SR Mehta, AJ Swamy. Current Trends in the Management of Typhoid Fever. JAFI;2003;59:130-5.

20. Babul Hasan, Sabera Gul Nahar, Laila Akter, Ahmed Abu Saleh. Antimicrobial sensitivity pattern of Salmonella typhi isolated from blood culture in a referral hospital. Bangladesh J Med Microbiol; 2011;5(1):16-20.

21. Harriet Ugboko and Nandita De. Mechanisms of Antibiotic resistance in Salmonella typhi. Int.J.Curr.Microbiol.App.Sci; 2014;3(12):461-76.

22. Chande C, Shrikhande S, Kapale S, Agrawal S and Fule RP. Change in antimicrobial resistance pattern of Salmonella Typhi in Central India. Indian J Med Res;2002;115:248-50.

23. Lovely Akter, Munir Hassan and Zakaria Ahmed. Present Status And Antibiotic Sensitivity Pattern Of Salmonella Typhi And S. Paratyphi In Different Age Group Hospitalized Patients In Dhaka City, Bangladesh. Journal of Pharmacy and Biological Sciences; 2012;4(3):27-30.

24. B. A. Rahman, M. O. Wasfy, M. A. Maksoud, N. Hanna, E. Dueger and B. House. Multi-drug resistance and reduced susceptibility to ciprofloxacin among Salmonella enterica serovar Typhi isolates from the Middle East and Central Asia. New Microbes and New Infections; 2014;2(4):88-92.

25. Antti Hakanen, Pirkko Kotilainen, Jari Jalava, Anja Siitonen, and Pentti Huovinen. Detection of Decreased Fluoroquinolone Susceptibility in Salmonellas and Validation of Nalidixic Acid Screening Test. Journal of Clinical Microbiology; 1999;37(11):3572-7.

26. Sadia Ikram, Shagufta Hussain, Ayesha Imtiaz, Muhammed Dilawar Khan. Nalidixic acid as a Predictor of Ciprofloxacin Susceptibility in Typhoid fever. Journal of Islamabad Medical and Dental College; 2015;4(3):118 21. 